\title{
Development of technical and economic systems in construction and their homeostatic balance
}

\author{
Sergey Sborshikov ${ }^{1}$, and Alexey Bobin ${ }^{1, *}$ \\ ${ }^{1}$ Moscow State University of Civil Engineering, Yaroslavskoe shosse, 26, Moscow, 129337, Russia
}

\begin{abstract}
Development of technical and economic systems, their direction and functionality is a continuous process of transition from one condition to another, includes random components, which got different impact on the trajectory of growth. In this work, we performed analysis of element of technical and economic system (construction sphere) at the level of the economic entity. Overcoming negative phenomena in the sustainable growth of technical and economic systems is closely connected with the successful solution of tasks in investment and construction sphere, based on an effective management.
\end{abstract}

\section{Introduction}

Homeostatic equilibrium reflects the essence of development of technical and economic systems at the levels of hierarchy in space and of the national economy. It also reflects the tendencies of the rapidly changing development systems. In modern conditions, the term of homeostatic equilibrium expresses the spatio-temporal state of coordination and synchronization of all interrelated processes in a generalized form.

The defining property of homeostatic equilibrium in the field of investment and construction sphere is its dynamic, rapidly changing character. The coordinates determining the state of equilibrium of the system at the moment, are under the influence of permanent and rapid changes that characterize the overall development of investment and construction sphere. Therefore, the state of homeostatic equilibrium is manifested through a general trend of constant adaptation to a variety of emerging impacts, through constant overcoming condition that have become undesirable due to scientific and technological progress. The balance-loss-balance ratio expresses dynamic changes in the state of development and its source in the field of investment and construction sphere.

The decisive condition for the optimal homeostatic balance is to ensure the proportionality of the development of all the elements functioning in the field of investment and construction sphere in their sectoral, corporate and regional context. Technical and economic systems are characterized by a complex structure. Their upward trajectory is the vector sum of exposure to many different economic factors. At the same time, homeostatic equilibrium state of the system corresponds to the internal local dynamic equilibrium

* Corresponding author: alexey.bobin@yandex.ru 
existing between its various constituent parts.

\section{Description of defining components}

To describe the general development of investment and construction sphere, it is not enough to know the direction, speed and acceleration of a single element, only a comprehensive examination of all the relationships that are formed in the technical and economic system and based on internal logic, opens up the opportunity to obtain information that might be suitable for effective management of production and supply processes.

The trajectory of the growth of investment and construction sphere represents itself the resultant of three values enabling to describe the direction of the technical and economic system (Figure 1).

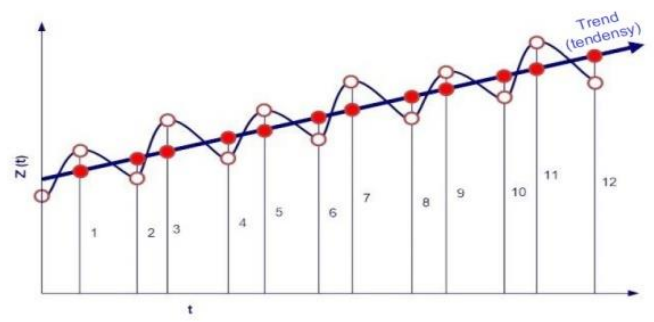

Fig. 1. Growth trajectory

Values:

- trend $q_{t}$

- oscillatory movement $\varphi_{t}$;

- random component $\varepsilon_{\mathrm{t}}$.

Therefore:

$$
z_{t}=q_{t}+\varphi_{t}+\varepsilon_{t} .
$$

The development of technical and economic systems, their direction and functionality is a continuous process of transition from one condition to another, higher qualitative and quantitative values.

Each state of the system represents a stage in the process of sustainable growth. Based on the trend that describes future development based on the previous states, the future state of the technical and economic system (investment and construction sphere) could be predicted at a certain level of the hierarchy.

Therefore, the trend is a way of expressing the main trend of the system development. It prevails over other features or tendencies, not relevant for the sustainable growth of the entire system. However, it should be noted that direction of the technical and economic system is equal to the interaction of objective laws of development and includes reasonable intervention of management structures.

While determining the trend based on averages represented as the sequence $\left\{z_{t}\right\}_{r_{-}}^{t}$, describing the development of the system from the initial time $t_{0}$ to the time $t_{p}$.

The quantitative formalization of the trend of long-term development of investment and construction sphere is that within a certain class of function $\mathrm{F}$ is determined the one that 
best reflects the growth of the system. It means that the function $f \in F$ needs to be found, where the values minimally deviate from the values of the state:

$$
\delta\left(f_{t}, z_{t}\right)=\min _{f_{r} \in F} \delta\left(f_{t}, z_{t}\right)
$$

if $\mathrm{t}=\mathrm{t}_{0}, \ldots, \mathrm{t}_{\mathrm{p}}$.

The specific calculations to find the difference between $z_{t}$ and $f_{t}$ can be done in different ways, for example:

$$
\begin{gathered}
\delta_{1}\left(f_{t}, z_{t}\right)=\sqrt{\sum_{t=t_{0}}^{t_{p}}\left(f_{t}-z_{t}\right)^{2}}, \\
\delta_{2}\left(f_{t}, z_{t}\right)=\sum_{t=t_{0}}^{t_{p}}\left|f_{t}-z_{t}\right| \text { or } \delta_{a}\left(f_{t}, z_{t}\right)=\max \left|f_{t}-z_{t}\right|, \\
t_{0} \leq t \leq t_{p} .
\end{gathered}
$$

The choice of F-class functions is based on researches on development rate of investment and construction sphere. If the increment is proportional to the achieved level, i.e. reveals the constantly growing rate, then the trend should be picked from the class of exponential functions. From the equation

$$
\mathrm{z}_{\mathrm{t}+1}-\mathrm{z}_{\mathrm{t}}=\alpha \mathrm{z}_{\mathrm{t}}
$$

Appears that

$$
z_{t}=(1+\alpha)^{t} z_{0} \text { if } t=1,2, \ldots
$$

According to this, the type of function below is selected:

$$
\mathrm{f}_{\mathrm{t}}=(1+\alpha)^{\mathrm{t}} \text {. }
$$

The $\alpha$ parameter is usually based on empirical values.In other situations the certain upper limit can be found, and then the increment at each time $t$ is proportional to the difference between the upper limit value of $\mathrm{z}^{*}$ and the already achieved level of $\mathrm{zt}$. In the form of an equation, it looks like:

$$
z_{t+1}-z_{t}=\beta\left(z^{*}-z_{t}\right)
$$

The solution of this equation looks like $z_{t}=z^{*}\left(1-b e^{-c t}\right)$, where $b=1-z_{0} / z_{x}$ and $c=-\log (1-\beta)$, and this solution is close to the $z_{\mathrm{t}}$ function only when values of $\mathrm{t}$ are high (or values of $b$ are very small).

This results in a function

$$
z_{\mathrm{t}}=\frac{z^{*}}{1+b e^{-c t}} .
$$

The function of this type describes the development trend of the system for the case of saturation. They can be described using the Thornquist function or the logarithmic function.

It is especially difficult to interpret the second component - oscillatory motion, which specifies the dynamics of investment and construction sphere as a technical and economic system. It is closely related to the specific features of development in the national economic field, and also depends on the strength and speed of ongoing production and support processes, as well as on the need to maintain a sustainable growth of investment and construction sphere.

The dynamic characters of the processes in construction, the constant changes in the 
conditions in which they are realized, define the nonlinear nature of their development.

The control subsystem provides the sustainable development of investment and construction sphere over time on the established growth paths, as well as compliance with the necessary proportions. The resultant of these complex effects in certain periods of time can lead to oscillatory motion relative to the state of the dynamic equilibrium (the amplitude of the oscillations is determined by specific conditions).

The growth trajectory of investment and construction sphere can be influenced by a number of factors associated with new technologies appearance, the growth of demand for certain types of construction products, differences between individual types and objects of construction in terms of productivity, consumption of material and technical resources. In these cases, the amplitude of the oscillatory motion is extinguished by the feedback and adequate management decisions in time. The presence of regulators that implement these solutions indicates that the dynamic equilibrium is also homeostatic.

Another factor causing the oscillatory movement is the interaction of investment and construction sphere and the national economy. In this connection, the trajectory of development systems can be influenced by conjunctural fluctuations in prices for raw and final materials, energy resources, an also for machinery and equipment.

Noncompliance with the terms of realization of contractual obligations for the construction products for various reasons causes failures that can spread wave-like at the levels of the hierarchy. Fluctuations can be caused by certain natural phenomena having the form of hindrances.

In the event of undesirable oscillatory motions, management decisions of regulators should enable to return technical and economic systems to the path of sustainable growth in the shortest possible time and with minimal costs. It should be noted that at the level of "construction site - building object" such effective regulator can be presented as the organizer of construction and a promising organizational management scheme implemented by him and based on engineering (engineering scheme). This scheme allows to adequately respond to permanent changes in the environment, to neutralize the negative manifestations of random factors.

As noted above, when technical and economic systems are moving along the path of sustainable development at different levels of the hierarchy, random factors arise that can cause rapid changes in investment and construction sphere. Such factors include, for example, the appearance of fundamentally new materials, machines, equipment and technologies.

In such situations, the trajectory of sustainable growth should take into account the possibility of identifying internal reserves, improving the organization of labor, rapid introduction of scientific ideas, product and technological innovations.

The identification of a random component in the development of investment and construction sphere plays an important role in predicting future conditions, as well as in determining the optimal parameters of the technical and economic system. In this regard, the task of the hierarchy level, at which investment and construction sphere is located, is to establish the area of possible trajectories and the probability of their implementation. This is one of the opportunities to justify decisions aimed at bringing investment and construction sphere closer to the path of sustainable growth.

Considering investment and construction sphere as a technical and economic system $\mathrm{S}$, which at the time $t$ is in the state of $z_{t}$, taking into account its dynamics it is possible to determine the set of states that can be achieved by the time $t+1$. We denote them as $\left\{z_{t+1}^{i}\right\}_{i-1}^{q}$, and the probability of transition from the state $z_{t}$ to the state $z_{t+1}^{i}$ is defined as $p_{t}^{i}$. This data fill the matrix $P_{z}^{S}$ : 


$$
P_{z_{t}}^{S}=\left(\begin{array}{ccc}
z_{t+1}^{1} & z_{t+1}^{2} \cdots & z_{t+1}^{q} \\
p_{t}^{1} & p_{t}^{2} \cdots & p_{t}^{q}
\end{array}\right) .
$$

The conditions must be met:

$$
p_{t}^{i} \geq 0_{\text {и }} \sum_{i=1}^{q} p_{t}^{i}=1
$$

If some probability of $p_{t}^{i}$ is equal to zero, it means that investment and construction sphere by the time $t+1$ cannot transform to the state $z_{t+1}^{i}$. In contrast, if a certain state has the probability $p_{t}^{i}=1$, the system will directly switch to that state at the time $t+1$. The probabilistic growth of the technical and economic system $\mathrm{S}$ at the time $\mathrm{t}$ is identified by a triple of indicators $\left\{\begin{array}{lll}t_{v} & z_{t}, & P_{z_{*}}^{s}\end{array}\right\}$

It should be pointed out that there is a close relationship between the concept of "probabilistic space for the growth of the technical and economic system" and the logistics concept of "achievable area". The probabilistic space of growth covers both the set of achievable states and the probability of their occurrence.

Knowledge of the structure of the probabilistic growth space of this technical and economic system is of particular importance for the prediction of investment and construction sphere.

In order to present the structure of the probabilistic space of growth of investment and construction sphere and to establish a set of trajectories of its growth, the entropy of the probabilistic space of growth is determined at the time $\mathrm{t}$ :

$$
H_{t}\left(P_{z_{t}}^{S}\right)=-\sum_{i=1}^{q} p_{t}^{i} \log _{2} p_{t}^{i}
$$

The entropy of the probabilistic growth space of the technical and economic system $\mathrm{S}$ indicates its capabilities in the complex process of sustainable development.

It should be noted that in the case where the probability of one of the possible states of the system is 1 , the entropy of its growth space takes its minimal value, i.e. zero.

The entropy of the probabilistic space of investment and construction sphere growth reaches its maximum if all probabilities $p_{t}^{i}$ are equal to each other, i.e.

$$
p_{t}^{1}=p_{t}^{2}=\cdots=p_{t}^{q}=\frac{1}{=}
$$

If the entropy of the probabilistic space of growth has a maximum value, it is a consequence of the fact that all the states of investment and construction sphere at the time $\mathrm{t}+1$ are equally possible.

Entropy characterizes the degree of uncertainty in the development of the technical and economic system. However, the concept of entropy can be used to describe accelerating (or vice versa gravitational) phenomena in the space of the national economy, and below in the hierarchy - the space of investment and construction sphere, etc.

\section{Growth process based on economic entities}

Within the framework of a single technical and economic system, there are certain elements (for example: construction sphere), the task of which is to accelerate the overall process of economic growth. Creation of regulated economic impacts, which are the driving force of the overall sustainable growth, in relation to investment and construction sphere is 
particularly important in planning and management in general, as well as in the design of development trajectories. Thus, the conditions are provided for the priority development of those elements of the system that accelerate its overall growth.

In order to present the accelerating or gravitational phenomena occurring in the space of investment and construction sphere, we will consider the technical and economic system at the level of the economic entity $\mathrm{S}^{*}$, which has an impact on the system $\mathrm{S}$. This is the case when the construction organizer contributes to the development of the contractor organization within the framework of effective investment and building activities based on the engineering management scheme. Thus, $\mathrm{S}^{*}$ is the organizer of the construction, and $\mathrm{S}$ is the contractor.

It is assumed that the system $\mathrm{S}^{*}$ at the time $\mathrm{t}$ is in the state $\mathrm{z}_{\mathrm{t}}$, and its economic space is characterized by a triple of indicators $\left\{\begin{array}{llll}t_{v} & z_{t_{*}} & P_{z_{*}}^{s^{*}}\end{array}\right\}$ with the number of states $\mathrm{n}$.

The system $\mathrm{S}$ can be in the states $y_{t+1}^{1}, y_{t+1}^{2}, \ldots, y_{t+1}^{i}$ and is described by $\left\{t, y_{t}, P_{v_{m}}^{s}\right\}$.

Since the growth process of the $\mathrm{S}$ system is associated with the development of the $\mathrm{S}^{*}$ system, the probability of its state growth also depend on the development of the $\mathrm{S}$ *system.

Thus, through

$$
\pi_{t}^{i k}=p_{t}\left(\frac{y_{t+1}^{i}}{z_{t+1}^{k}}\right)
$$

let us denote a conditional probability, i.e. $\pi_{t}^{i k}$ is the probability that the system $\mathrm{S}$ will be in the state $y_{t+1}^{i}$ at the time $t+1$, provided that the system $S^{*}$ is in the state $z_{t+1}^{k}$.

The entropy of the probabilistic growth space of the technical and economic system $\mathrm{S}$ in the case when the system $S^{*}$ at the time $t+1$ is in the state $z_{t+1}^{\mathbb{l}}$, is calculated by the formula

$$
H_{t}^{\mathbb{l}}\left(P_{y_{\mathrm{t}}}^{s}\right)=-\sum_{\mathrm{i}=1}^{\mathbb{l}} \pi_{t}^{\mathrm{ill}} \lg _{g_{2}} \pi_{t}^{\mathrm{il}} .
$$

Since the activity of the construction organizer as a system $\mathrm{S}^{*}$ can be at the time $t+1$ in each of its possible states $z_{t+1}^{\mathbb{1}}$, with the probability $p_{t}^{i}$, it is necessary to consider the whole set of these probabilities.

In order to quantify the influence that the growth of the leading system $\mathrm{S}^{*}$ has on the development of the system $\mathrm{S}$, we introduce the entropy of the probabilistic space of growth of the system $\mathrm{S}$, depending on the development of the leading system $\mathrm{S}^{*}$. It is calculated as follows:

$$
H_{t}\left(P_{v_{*}}^{s} \mid P_{z_{*}}^{s^{*}}\right)=-\sum_{i=1}^{n} p_{*}^{j} H_{*}^{j}\left(P_{v_{*}}^{s}\right)=\sum_{i=1}^{n} \sum_{i=1}^{\|} p_{*}^{j} \pi_{*}^{i j} l g_{2} \pi_{*}^{i j} .
$$

Between the entropy of the probabilistic space of growth of the system $\mathrm{S}^{*}$ and the entropy of the system $\mathrm{S}$ under the condition of the action of the system $\mathrm{S}^{*}$, there is such a relation:

$$
H_{\mathrm{t}}\left(P_{y_{\mathrm{t}}}^{s} \mid P_{z_{\mathrm{t}}}^{s^{*}}\right) \leq H_{\mathrm{t}}\left(P_{y_{\mathrm{t}}}^{s}\right) .
$$

Equality occurs only if the growth of the technical and economic system $S^{*}$ does not affect the development of the system $\mathrm{S}$. 
a)

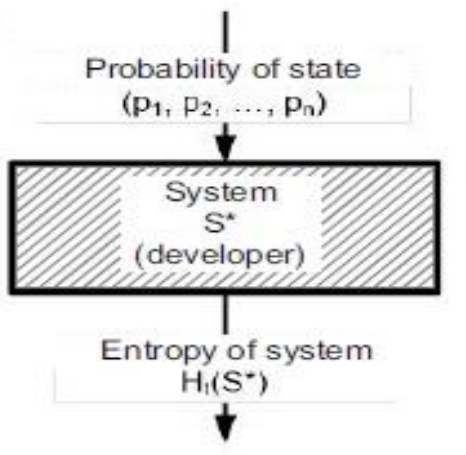

б)

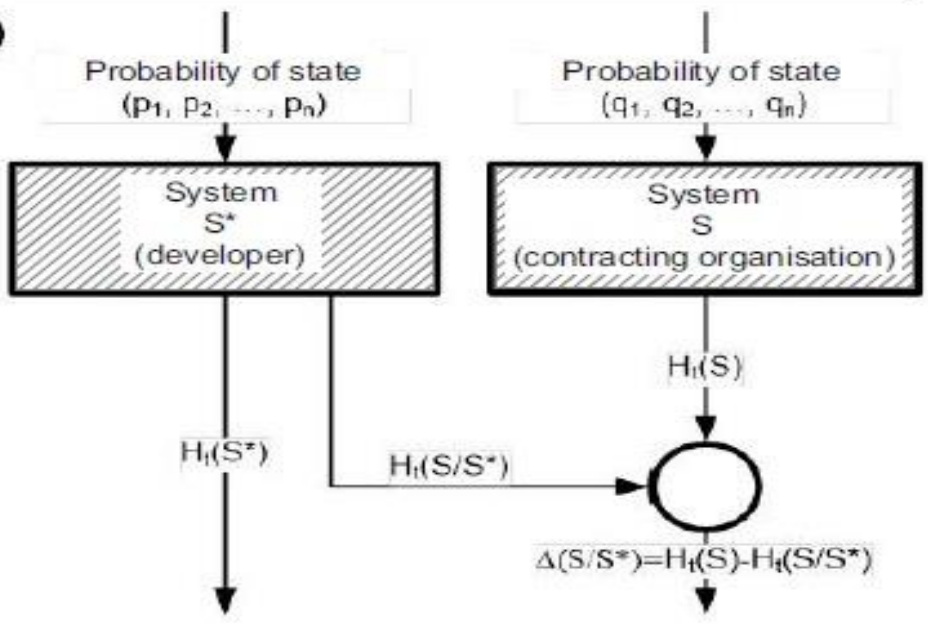

Fig. 2. Entropy of technical and economic systems: a - isolated systems; b - connected systems

The influence of the $\mathrm{S}^{*}$ system (construction organizer) on the growth dynamics of the $\mathrm{S}$ system (contractor) is shown in Figure 2 and is quantified by the difference

$$
\Delta_{\mathrm{t}}\left(P_{v_{*}}^{S} \mid P_{z_{*}}^{5^{*}}\right)=H_{\mathrm{t}}\left(P_{v_{*}}^{S}\right)-H_{\mathrm{t}}\left(P_{v_{*}}^{S} \mid P_{z_{*}}^{S^{*}}\right) \text {. }
$$

If we assume that the system $\mathrm{S}^{*}$ (organizer of construction) has a positive impact on the development of systems $S_{1}, S_{2}, \ldots, S_{N}$ (contractors) within the framework of the engineering scheme management of investment and construction sphere, then quantitatively this effect, i.e. the gravitational force of the system $\mathrm{S}^{*}$, is expressed by the characteristic

$$
F_{t}\left(P_{z_{*}}^{S^{*}}\right)=\sum_{k=1}^{N} \Delta_{t}\left(P_{v_{*}}^{S_{k}} \mid P_{z_{*}}^{S^{*}}\right) \text {. }
$$

A special problem related to the verification of the properties of motion and sustainable development of technical and economic systems at the levels of hierarchy concerns the entropy of the entire region of growth trajectories.

Consider another case, investment and construction sphere as a technical and economic system $\mathrm{S}$, which is in the state of $\mathrm{z}_{\mathrm{t}}$. Its growth probability space at time $\mathrm{t}$ is $\left\{\begin{array}{lll}t_{v} & z_{\mathrm{t}}, & P_{z_{*}}^{s}\end{array}\right\}$, 
and the entropy is $H_{t}\left(P_{z_{*}}^{s}\right)$. By the time $\mathrm{t}+2$ the system can be in the states $z_{t+2}^{1}, z_{t+2}^{2}, \ldots, z_{t+2}^{q}$, the probabilities of which are reflected in the following matrix:

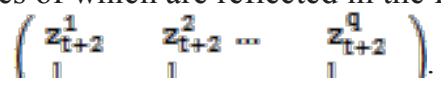

The above probabilities depend on the state of investment and construction sphere at the time $\mathrm{t}+1$. Thus is revealed the necessity to take into account the probability like $p\left(z_{t+2} \mid z_{t+1}\right)$.

They indicate the probability with which investment and construction sphere will be in the state $z_{t+2}$ at the time $t+2$ if at the time $t+1$ it was in the state $z_{t+1}$.

The entropy of the probability space of sustainable growth of investment and construction sphere at the time $t+2$, which is due to its state at the time $t+1$, is described as follows:

$$
H_{t+1}\left(P_{z_{t+2}}^{S} \mid P_{z_{t+1}}^{S}\right)=-\sum_{k=1}^{q} p_{k} H_{t+1}^{k} \cdot\left(P_{z_{t+2}}^{S}\right)
$$

$H_{t+1}^{k}\left(P_{z+1}^{S}\right)$ is the entropy of the probabilistic space of investment and construction sphere at the time $t+2$, if at the time $t+1$ it was in the state $z_{t+1}^{k}$.

\section{Discussion}

Knowledge of the probabilistic space of investment and construction sphere growth and identification of entropy can be a useful tool for practical forecasting and construction planning, as well as for the management of systems at hierarchy levels. It also describes processes of accelerating (or gravitational) phenomena in the space of the national economics, and determinate the way of it development. Also, as promising areas for further scientific research, it is possible to specify:

1. Methodological foundations determining ways to decrease the negatve impact of random component.

2. To improve the scientific and methodological basis for the procedure of management decision-making.

\section{References}

1. S.B. Sborshikov, I M Markova, Bulletin MGSU 4-5 (2010)

2. A.A. Volkov, S. B. Sborshikov, T. V. Khripko, Bulletin MGSU 10 (2016)

3. T.V. Prokhorova, Natural and technical sciences 6 (2015)

4. S.B. Sborshikov, D. M. Leybman, Scientific Review, 18 (2016)

5. N.V. Lazareva, Estimated contract work in construction, 3 (2016)

6. P.A. Zhuravlev, S.B. Sborshikov, Bulletin of the Belgorod STU 198 (2017)

7. Y.V. Zharov Scientific Review, 432 (2015)

8. Y.V. Zharov, Scientific Review, 242 (2015)

9. A. A. Bobin, Estimated contract work in construction 46 (2016) 